\title{
PRACTICAL LYAPUNOV STABILITY CRITERIA FOR DIFFERENTIAL ALGEBRAIC EQUATIONS
}

\author{
ROSWITHA MÄRZ \\ Humboldt-Universität zu Berlin, Fachbereich Mathematik \\ Unter den Linden 6, 10099 Berlin, Germany
}

1. Introduction. Consider quasilinear differential algebraic equations (DAEs)

$$
A x^{\prime}(t)+g(x(t))=0
$$

where the leading coefficient matrix $A \in L\left(\mathbb{R}^{m}\right)$ is singular and $g: \mathcal{D} \rightarrow \mathbb{R}^{m}$ is $C^{1}$, $\mathcal{D} \subseteq \mathbb{R}^{m}$ open. Those DAEs are well known to arise in describing the dynamics of circuits, chemical reactions subject to invariants, constrained dynamical systems etc. No doubt, stability criteria that can also be checked numerically would be welcome even in those fields of applications.

From a geometric viewpoint, (1.1) should induce a smooth vector field on a certain state manifold. However, if it does, the vector field as well as the manifold are given only implicitly, and they are not available in practice for higher index DAEs except for interesting case studies.

Further, it should be mentioned that viewing a DAE as a differential equation on a manifold (see e.g. [8]) requires more smoothness than seems to be natural.

For instance, the so-called index 2 Hessenberg form DAE

$$
u^{\prime}-g(u, v)=0, \quad h(u)=0
$$

leads to the vector field

$$
\begin{aligned}
& u^{\prime}=g(u, v), \\
& v^{\prime}=-\left(h^{\prime}(u) g_{v}^{\prime}(u, v)\right)^{-1}\left\{h^{\prime}(u) g_{u}^{\prime}(u, v)+h^{\prime \prime}(u) g(u, v)\right\} g(u, v)
\end{aligned}
$$

on the manifold

$$
\mathcal{M}_{2}:=\left\{\left(u^{\mathrm{T}}, v^{\mathrm{T}}\right)^{\mathrm{T}} \in \mathbb{R}^{m}: h(u)=0, h^{\prime}(u) g(u, v)=0\right\}
$$

where the matrix $h^{\prime}(u) g_{v}^{\prime}(u, v)$ is assumed to be nonsingular.

1991 Mathematics Subject Classification: 34A08, 34D20, 34A10.

The paper is in final form and no version of it will be published elsewhere. 
For given $\left(u_{0}^{\mathrm{T}}, v_{0}^{\mathrm{T}}\right)^{\mathrm{T}} \in \mathcal{M}_{2}$, system (1.2) is shown to have a solution $u \in C^{1}$, $v \in C$, passing through that point (cf. [7]) while the analogous initial value problem (IVP) for (1.3) requires some more smoothness.

Moreover, considering the differential equation (1.3) on the whole space $\mathbb{R}^{m}$ instead of on the manifold $\mathcal{M}_{2}$ would not be helpful, since this so-called underlying regular ordinary differential equation (ODE) might show a completely different stability behaviour than (1.2) and (1.3) on $\mathcal{M}_{2}$.

In this paper we try to transfer classical results concerning Lyapunov stability of stationary solutions of regular ODEs to the case of DAEs (1.1), keeping smoothness as low as possible. For equilibrium points $x_{*} \in \mathcal{D}$ of (1.1), we formulate stability criteria in terms of the matrices $A, g^{\prime}\left(x_{*}\right)$ only.

As a by-product, we prove certain new solvability statements for index 3 equations (1.1) as well as for index 1 equations

$$
A(x(t)) x^{\prime}(t)+g(x(t))=0
$$

where the leading coefficient matrix $A(x)$ has an $x$-dependent null space.

The paper is organized as follows: In Section 2 we collect the necessary background material, report on index 1 and index 2 results, and apply them to equation (1.4). Section 3 deals with solvability and asymptotical stability for index 3 DAEs. The results obtained are then specified for the case of constrained multibody systems. The Appendix contains some facts we need on matrix calculus.

2. Index 1 and index 2 cases. Let $Q \in L\left(\mathbb{R}^{m}\right)$ denote any projector onto the null space $\operatorname{ker}(A)=: N$ of the leading coefficient $A$, and let $P:=I-Q$. Because of $A=A P$, equation (1.1) may be rewritten as

$$
A(P x)^{\prime}(t)+g(x(t))=0 .
$$

Now we agree to accept continuous functions $x: \mathcal{I} \rightarrow \mathbb{R}^{m}$ with continuously differentiable components $P x$, which satisfy (2.1) on the interval $\mathcal{I} \subseteq \mathbb{R}$, to be solutions of (1.1). Denote the related function space by

$$
C_{N}^{1}\left(\mathcal{I}, \mathbb{R}^{m}\right):=\left\{x \in C\left(\mathcal{I}, \mathbb{R}^{m}\right): P x \in C^{1}\left(\mathcal{I}, \mathbb{R}^{m}\right)\right\},
$$

or briefly by $C_{N}^{1}$.

Trivially, all orbits belong to the set

$$
\mathcal{M}_{1}:=\{w \in \mathcal{D}: g(w) \in \operatorname{im}(A)\},
$$

i.e., if $x \in C_{N}^{1}$ solves the DAE (1.1), then

$$
x(t) \in \mathcal{M}_{1}, \quad t \in \mathcal{I} .
$$

The best understood class of DAEs is that of index 1 equations, for which $\mathcal{M}_{1}$ becomes the set of all consistent initial values, that is, a solution passes through each $x_{0} \in \mathcal{M}_{1}$, meaning that $\mathcal{M}_{1}$ becomes the state manifold. 
Definition. The DAE (1.1) is called an index 1 tractable (or a transferable) $\mathrm{DAE}$ on $\mathcal{D}$ if the decomposition

$$
S_{0}(x) \oplus N=\mathbb{R}^{m}, \quad x \in \mathcal{D},
$$

holds, where

$$
S_{0}(x):=T_{x} \mathcal{M}_{1}=\left\{z \in \mathbb{R}^{m}: g^{\prime}(x) z \in \operatorname{im}(A)\right\}
$$

is the tangent space of $\mathcal{M}_{1}$ at $x \in \mathcal{M}_{1}$.

R e m a rks. 1. An equivalent formulation of (2.3) is the following: The matrix pencil $\left\{A, g^{\prime}(x)\right\}$ is regular, and index $\left\{A, g^{\prime}(x)\right\}=1, x \in \mathcal{D}$. This is the origin of the notion of an "index 1 DAE" (coming from [2]).

2. Put $B:=g^{\prime}\left(x_{0}\right)$ for given $x_{0} \in \mathcal{M}_{1}$. Then, by Lemma 4.1 below, $A+B Q=$ $A_{1}$ is nonsingular. Define $u_{0}:=P x_{0}, v_{0}:=Q x_{0}$; further, let $\bar{u}_{0} \in \operatorname{im}(P)$ be determined by $g\left(u_{0}+v_{0}\right)=-A \bar{u}_{0}$. Consider $F(w, u):=A w+g(u+Q w)$ on a neighbourhood of $\left(w_{0}, u_{0}\right), w_{0}:=\bar{u}_{0}+v_{0}$. Clearly, $F\left(w_{0}, u_{0}\right)=0, F_{w}^{\prime}\left(w_{0}, u_{0}\right)=$ $A_{1}$, so that the Implicit Function Theorem provides a $C^{1}$ function $w=f(u)$, $F(f(u), u)=0$. Solving the IVP

$$
u^{\prime}(t)+P A_{1}^{-1} g(u(t)+Q f(u(t)))=0, \quad u\left(t_{0}\right)=u_{0}=P x_{0}
$$

and putting

$$
v(t):=Q f(u(t))
$$

we may easily check $x(t):=u(t)+v(t)$ to be the only solution of (1.1) satisfying

$$
x\left(t_{0}\right)=x_{0} \in \mathcal{M}_{1} \text {. }
$$

Hence, the IVP (1.1), (2.7) is locally, equivalently transferred into the state variable form (2.5), (2.6), which leads to the notion of a "transferable DAE" (cf. [3]).

3. Since $f$ is $C^{1}$, so is $v$. Consequently, solutions of an index 1 DAE (1.1) are $C^{1}$ solutions in fact.

4. Obviously, also the IVPs for (1.1) with the initial condition

$$
P\left(x(t)-x^{0}\right)=0, \quad x^{0} \in \mathbb{R}^{m},\left|P x^{0}-P x_{0}\right| \text { sufficiently small },
$$

do have solutions

$$
x\left(t ; x^{0}, t_{0}\right)=u\left(t ; P x^{0}, t_{0}\right)+Q f\left(u\left(t ; P x^{0}, t_{0}\right)\right),
$$

but now the related consistent initial value is $x\left(t_{0} ; x^{0}, t_{0}\right)=P x^{0}+Q f\left(P x^{0}\right)$. In general, we expect that $x\left(t_{0} ; x^{0}, t_{0}\right) \neq x^{0}$ since we have not tried to choose $x^{0} \in \mathcal{M}_{1}$.

5. Obviously, we can apply the above definition to each open subset $U \subset \mathcal{D}$ instead of $\mathcal{D}$ itself.

THEOREM 2.1. Let $g$ be a $C^{2}$ function, and $x_{*} \in \mathcal{D}$ be an equilibrium point of (1.1), i.e. $g\left(x_{*}\right)=0$. Let $\left\{A, g^{\prime}\left(x_{*}\right)\right\}$ be a regular index 1 pencil, and let all its eigenvalues have negative real parts. Then there are $a \tau>0$ and $a \delta(\varepsilon)>0$ for each $\varepsilon>0$ such that 

$\left[t_{0}, \infty\right)$

(i) all IVPs (1.1), (2.8), $\left|P x^{0}-P x_{*}\right| \leq \tau$ have unique solutions defined on

(ii) $\left|P x^{0}-P x_{*}\right| \leq \delta(\varepsilon)$ implies $\left|x\left(t ; x^{0}, t_{0}\right)-x_{*}\right| \leq \varepsilon, t \geq t_{0}$, and

(iii) $\left|x\left(t ; x^{0}, t_{0}\right)-x_{*}\right| \rightarrow 0(t \rightarrow \infty)$.

Proof. Without loss of generality we may assume $Q$ to project onto $N$ along $S\left(x_{*}\right)$.

Define $B:=g^{\prime}\left(x_{*}\right)$. Clearly, the matrix $M:=-P A_{1}^{-1} B$ is responsible for the stability behaviour of the ODE given by (2.5).

By Lemma 4.1, we have $M=P M=M P, Q=Q A_{1}^{-1} B$.

Suppose $(\lambda A+B) w=0, w \neq 0$. Multiplying by $P A_{1}^{-1}$ and $Q A_{1}^{-1}$ we decouple this equation into

$$
(\lambda P-M) w=0, \quad Q w=0 .
$$

Therefore, all eigenvalues of the pencil are at the same time eigenvalues of $M$. On the other hand, each nontrivial eigenvalue of $M$ belongs also to the pencil spectrum, since $M z=\lambda z, \lambda \neq 0, z \neq 0$ implies $z=P z, A_{1}^{-1} B z-Q A_{1}^{-1} B z+\lambda z$ $=0$, thus $B z+\lambda A_{1} P z=B z+\lambda A z=0$. Additionally, $M$ has the zero eigenvalue, the related eigenvectors $\operatorname{span} \operatorname{ker}(A)=\operatorname{ker}(M)$.

Finally, by slightly modified standard arguments (cf. [7], Lemma 4.4), which take into account that we are interested in initial values for (2.5) belonging to $\operatorname{im}(P)$ only, the statements of the theorem may be obtained.

Rem arks. 1. Theorem 2.1 generalizes the classical Lyapunov Theorem (with $\left.A=I, P=I, \mathcal{M}_{1}=\mathcal{D}\right)$. Note that the assumption for $g$ to be $C^{2}$ is standard there.

2. Note that $A_{1}(x):=A+g^{\prime}(x) Q$ remains nonsingular for $x$ from a neighbourhood $U \subseteq \mathcal{D}$ of $x_{*}$, since so is $A_{1}\left(x_{*}\right)=A+B Q$. This means that (1.1) is an index 1 DAE on $U$.

Due to Theorem 2.1, in order to be sure that an equilibrium is asymptotically stable it will do to check the spectrum of the pencil $\left\{A, g^{\prime}\left(x_{*}\right)\right\}$ only. Moreover, if the DAE (1.1) itself is not known to have index 1 in advance, it is sufficient to compute index 1 for the pencil formed at this single point.

Unfortunately, for higher index DAEs the situation is more complicated. On the one hand, the state manifold is now a submanifold of $\mathcal{M}_{1}$ only. This fact is illustrated e.g. by example (1.2), where we would have $\mathcal{M}_{1}:=\left\{\left(u^{\mathrm{T}}, v^{\mathrm{T}}\right)^{\mathrm{T}} \in \mathbb{R}^{m}\right.$ : $h(u)=0\}$, but $\mathcal{M}_{2}$ given in $\S 1$ is the state manifold. On the other hand, the pencil $\left\{A, g^{\prime}\left(x_{0}\right)\right\}$ given at a single point $x_{0}$ only does not contain sufficient information for determining the index. This will be demonstrated by example (2.12) below.

Definition. The DAE (1.1) is called index 2 tractable on $\mathcal{D}$ if

$$
\begin{gathered}
S_{0}(x) \cap N \neq\{0\}, \\
S_{1}(x) \oplus N_{1}(x)=\mathbb{R}^{m}, \quad x \in \mathcal{D},
\end{gathered}
$$


where

$$
S_{1}(x):=\left\{z \in \mathbb{R}^{m}: g^{\prime}(x) P z \in \operatorname{im}\left(A_{1}(x)\right)\right\}, \quad A_{1}(x):=A+g^{\prime}(x) Q,
$$

and the null space $N_{1}(x):=\operatorname{ker}\left(A_{1}(x)\right)$ has constant dimension (see e.g. [5], [7]).

Remarks. 1. Note that the conditions (2.9), (2.10) are satisfied if and only if $\left\{A, g^{\prime}(x)\right\}$ is a regular index 2 pencil for all $x \in D$ ([5], Theorem 2.6).

2 . Dimension changes of the null space $N_{1}(x)$ may give rise to new singularities like bifurcations etc. (cf. also example (2.12) below).

3. By Lemma 4.1 below, the condition (2.10) is satisfied if and only if the matrix

$$
G_{2}(x):=A_{1}(x)+g^{\prime}(x) P Q_{1}(x)
$$

remains nonsingular for all $x \in \mathcal{D}$, where $Q_{1}(x) \in L\left(\mathbb{R}^{m}\right)$ denotes any projector onto $N_{1}(x)$.

4. Again we can apply our definition to an open subset $U \subset \mathcal{D}$ instead of $\mathcal{D}$.

5. It may be shown that

$$
\mathcal{M}_{2}=\left\{w \in \mathcal{M}_{1}: g^{\prime}(w) P A^{+} g(w) \in \operatorname{im}\left(A_{1}(w)\right)\right\}
$$

describes the state manifold of an index 2 DAE (1.1), where $A^{+}$denotes the Moore-Penrose inverse of $A$. However, formula (2.11) seems to be of no practical use.

6. Recall that index 1 DAEs were characterized by nonsingular $A_{1}(x)$, that is, we obtain $\mathcal{M}_{2}=\mathcal{M}_{1}$ when formally computing $\mathcal{M}_{2}$ for an index 1 equation.

The problem with testing index 2 at a single point is the following: Suppose that, at some point $x_{0}$, we know $A_{1}\left(x_{0}\right)$ to be singular, but $G_{2}\left(x_{0}\right)$ nonsingular. Clearly, for $x$ belonging to a neighbourhood of $x_{0}, G_{2}(x)$ remains nonsingular provided $Q_{1}(x)$ is continuous. However, $A_{1}(x)$ may become nonsingular for $x \neq x_{0}$ or change the null space dimension.

Let us illustrate this situation by the following example (cf. [1], [7]), which describes a simple nonlinear resistor circuit. For the system

$$
\left\{\begin{aligned}
x_{1}^{\prime}-\alpha\left(x_{3}\right) & =0, \\
x_{2}^{\prime}-\beta\left(x_{3}\right) & =0, \\
x_{3}^{3}+x_{2} x_{3}+x_{1} & =0
\end{aligned}\right.
$$

with smooth given functions $\alpha, \beta: \mathbb{R} \rightarrow \mathbb{R}$, we compute

$$
A_{1}(x)=\left[\begin{array}{ccc}
1 & 0 & -\alpha^{\prime}\left(x_{3}\right) \\
0 & 1 & -\beta^{\prime}\left(x_{3}\right) \\
0 & 0 & x_{2}+3 x_{3}^{2}
\end{array}\right], \quad P=A=\left[\begin{array}{ccc}
1 & 0 & 0 \\
0 & 1 & 0 \\
0 & 0 & 0
\end{array}\right] \text {; }
$$

further,

$$
\begin{aligned}
N & =\left\{z \in \mathbb{R}^{3}: z_{1}=z_{2}=0\right\}, \\
S_{0}(x) & =\left\{z \in \mathbb{R}^{3}: z_{1}+x_{3} z_{2}+\left(x_{2}+3 x_{3}^{2}\right) z_{3}=0\right\}, \\
\mathcal{M}_{1} & :=\left\{w \in \mathbb{R}^{3}: w_{1}+w_{2} w_{3}+w_{3}^{3}=0\right\} .
\end{aligned}
$$


Trivially, all solutions of (2.12) lie within the set $\mathcal{M}_{1}$, describing a surface that has a fold. Denote by $H:=\left\{x \in \mathcal{M}_{1}: x_{2}+3 x_{3}^{2}=0\right\}$ the set of points belonging to the fold curves.

Clearly, (2.12) has index 1 on $\mathbb{R}^{3} \backslash\left\{x \in \mathbb{R}^{3}: x_{2}+3 x_{2}^{2}=0\right\}$, and there are unique solutions passing through the points $x_{0} \in \mathcal{M}_{1} \backslash H$ at time $t_{0}$. However, on $H$ the situation changes considerably, since $A_{1}(x)$ becomes singular there. Formally, we have

$$
\begin{aligned}
N_{1}(x) & =\left\{z \in \mathbb{R}^{3}: z_{1}-2 x_{3} z_{3}=0, z_{2}=z_{3}\right\}, \\
S_{1}(x) & =\left\{z \in \mathbb{R}^{3}: z_{1}+x_{3} z_{2}=0\right\}=S_{0}(x) \quad \text { for } x \in H,
\end{aligned}
$$

hence $N_{1}(x) \cap S_{1}(x)=\{0\}$ for all $x \in H \backslash\{0\}$, thus all related pencils $\left\{A, g^{\prime}(x)\right\}$ are regular of index 2. Different choices of $\alpha, \beta$ lead to bifurcations and impasse points (cf. [1], [7]). In the trivial case of $\alpha\left(x_{3}\right) \equiv \beta\left(x_{3}\right) \equiv 0$, all solutions are stationary.

On the whole, the DAE (2.12) represents a rather singular index 1 DAE (with singularities on $H)$. Obviously, checking only the pencil $\left\{A, g^{\prime}\left(x_{0}\right)\right\}$ for some $x_{0} \in$ $H$ would not provide sufficient information on the DAE.

To avoid those singularities we restrict the class of DAEs. By [7], Lemma 2.2, for a large class of DAEs, considering a single pencil $\left\{A, g^{\prime}\left(x_{0}\right)\right\}$ will do.

Lemma 2.2. Let $R \in L\left(\mathbb{R}^{m}\right)$ denote any projector onto $\operatorname{im}(A)$. For given $x_{0} \in \mathcal{D}$, with $B:=g^{\prime}\left(x_{0}\right)$, let the matrix pencil $\{A, B\}$ be regular with index 2 . Suppose

$$
(I-R)\{g(y)-g(P y)\} \in \operatorname{im}((I-R) B Q), \quad y \in U_{0},
$$

where $U_{0} \subset \mathcal{D}$ denotes a neighbourhood of $x_{0}$. Then the DAE (1.1) is index 2 tractable on a neighbourhood $U \subseteq U_{0}$ of $x_{0}$.

For the proof we refer to [7].

Note that condition (2.13) means, roughly speaking, that the derivative free part $(I-R) g(x)$ in (1.1) should depend on the component $Q x$ only linearly. The Hessenberg form DAE (1.2) has this property trivially.

TheOREM 2.3. Let $g: \mathcal{D} \rightarrow \mathbb{R}^{m}$ be of class $C^{1}$, and let the assumptions of Lemma 2.2 be valid. Additionally, let $Q_{1}, P_{1}, A_{2} \in L\left(\mathbb{R}^{m}\right)$ be determined by Lemma 4.2 below, and let the consistency conditions

$$
g\left(x_{0}\right) \in \operatorname{im}(A), \quad Q A_{2}^{-1} g\left(x_{0}\right)=0
$$

be satisfied. Then

(i) There is a $\tau>0$ so that all IVPs for (1.1) completed by the initial condition

$$
\begin{gathered}
P P_{1} x\left(t_{0}\right)=P P_{1} x^{0}, \quad x^{0} \in \mathbb{R}^{m}, \\
\left|P P_{1} x^{0}-P P_{1} x_{0}\right| \leq \tau,
\end{gathered}
$$

are solvable in $C_{N}^{1}$. 
(ii) If, additionally, $Q_{1} A_{2}^{-1} g$ is of class $C^{2}$ then these IVPs are uniquely solvable, and the solutions belong to the class $C^{1}$.

For the proof we refer to [7]. Recall only that the basic idea is to use the decoupling of the linear part of

$$
A x^{\prime}(t)+B x(t)+h(x(t))=0,
$$

where $h(y):=g(y)-g^{\prime}\left(x_{0}\right) y$.

The inherent local state equation is of type

$$
\begin{gathered}
u^{\prime}+P P_{1} A_{2}^{-1} B u+P P_{1} A_{2}^{-1} h(\varphi(u))=0, \\
u\left(t_{0}\right)=P P_{1} x^{0} .
\end{gathered}
$$

Theorem 2.4. Let $g: \mathcal{D} \rightarrow \mathbb{R}^{m}$ be of class $C^{2}, x_{*} \in \mathcal{D}, g\left(x_{*}\right)=0$; further, let condition (2.13) be valid on a neighbourhood $U_{0}$ of $x_{*}$. Let the pencil $\left\{A, g^{\prime}\left(x_{*}\right)\right\}$ be regular of index 2 , and let all its eigenvalues have negative real parts. Then there are $\tau>0$ and $\delta(\varepsilon)>0$ for each $\varepsilon>0$ such that

(i) all IVPs (1.1), (2.15), $\left|P P_{1}\left(x^{0}-x_{*}\right)\right| \leq \tau$ have unique solutions defined on $\left[t_{0}, \infty\right)$,

(ii) $\left|P P_{1} x^{0}-P P_{1} x_{*}\right| \leq \delta(\varepsilon)$ implies $\left|x\left(t ; x^{0}, t_{0}\right)-x_{*}\right| \leq \varepsilon$ for all $t \geq t_{0}$, and

(iii) $\left|x\left(t ; x^{0}, t_{0}\right)-x_{*}\right| \rightarrow 0(t \rightarrow \infty)$.

The proof is carried out in [7]. There, due to Lemma 4.4 below, a Lyapunov function related to $\operatorname{im}\left(P P_{1}\right)$ is used to continue the local solutions provided by Theorem 2.3 and the locally inherent regular ODE (2.16).

Next we apply the above results to the equation

$$
A(x(t)) x^{\prime}(t)+g(x(t))=0,
$$

where the matrix function $A: \mathcal{D} \rightarrow L\left(\mathbb{R}^{m}\right)$ is also assumed to belong to $C^{1}$.

If $A(y)$ has a constant null space, i.e.

$$
\operatorname{ker}(A(y))=: N, \quad y \in \mathcal{D},
$$

then we turn to the enlarged system

$$
\left\{\begin{aligned}
P x^{\prime}-y & =0, \\
A(x) y+g(x) & =0,
\end{aligned}\right.
$$

where $P=I-Q$, and $Q$ denotes a projector onto $N$ as above.

If the null space of $A(y)$ depends on $y$, then we turn to

$$
\left\{\begin{aligned}
x^{\prime}-y & =0, \\
A(x) y+g(x) & =0 .
\end{aligned}\right.
$$

Both enlarged systems (2.18) and (2.19) have the form (1.1), i.e.

$$
\widetilde{A} \widetilde{x}^{\prime}(t)+\widetilde{g}(\widetilde{x}(t))=0 .
$$


In the first case, index 1 tractability (transferability) is defined (cf. e.g. [3]) as described above by the use of

$$
A(x) \quad \text { and } \quad A_{x}^{\prime}(x) y+g^{\prime}(x)
$$

instead of $A$ and $g^{\prime}(x)$. In particular, the "transferability matrix" is now (on account of $\left.A_{x}^{\prime}(x) y Q=0\right)$

$$
A_{1}(x)=A(x)+g^{\prime}(x) Q .
$$

It is well known that (2.18) is index 1 tractable on $\mathcal{D} \times \mathbb{R}^{m}$ if and only if $(2.17)$ is index 1 tractable on $\mathcal{D}$. For given $x_{0} \in \mathcal{D}, y_{0} \in \mathbb{R}^{m}$, the related matrices for (2.18) are

$$
\begin{aligned}
\widetilde{P} & =\widetilde{A}=\left[\begin{array}{ll}
P & 0 \\
0 & 0
\end{array}\right], \quad \widetilde{Q}=\left[\begin{array}{ll}
Q & 0 \\
0 & I
\end{array}\right], \\
\widetilde{B} & =\left[\begin{array}{cc}
0 & -I \\
A_{x}^{\prime}\left(x_{0}\right) y_{0}+g^{\prime}\left(x_{0}\right) & A\left(x_{0}\right)
\end{array}\right], \\
\widetilde{A} & =\widetilde{A}+\widetilde{B} \widetilde{Q}=\left[\begin{array}{cc}
P & -I \\
g^{\prime}\left(x_{0}\right) Q & A\left(x_{0}\right)
\end{array}\right], \\
\widetilde{A}_{1}^{-1} & =\left[\begin{array}{cc}
P & A_{1}^{-1}\left(x_{0}\right) \\
-Q & P A_{1}^{-1}\left(x_{0}\right)
\end{array}\right], \\
\widetilde{M} & =-\widetilde{P} \widetilde{A}_{1}^{-1} \widetilde{B}=-\left[\begin{array}{cc}
P A_{1}^{-1}\left(A_{x}^{\prime} y_{0}+g^{\prime}\left(x_{0}\right)\right) & 0 \\
0 & 0
\end{array}\right] .
\end{aligned}
$$

Hence, Theorem 2.1 can be applied immediately.

In the second case, if $\operatorname{ker}(A(y))$ depends on $y$, we may formally extend the notion "index 1" via the transferability matrix

$$
A(x)+\left(A_{x}^{\prime}(x) y+g^{\prime}(x)\right) Q(x)=: A_{1}(x, y)
$$

where $Q(x)$ is again a projector onto $\operatorname{ker}(A(x))$. Now we derive, for given $x_{0} \in \mathcal{D}$, $y_{0} \in \mathbb{R}^{m}$

$$
\widetilde{P}=\widetilde{A}=\left[\begin{array}{ll}
I & 0 \\
0 & 0
\end{array}\right], \quad \widetilde{Q}=\left[\begin{array}{ll}
0 & 0 \\
0 & I
\end{array}\right], \quad \widetilde{A}_{1}=\left[\begin{array}{cc}
I & -I \\
0 & A\left(x_{0}\right)
\end{array}\right] .
$$

$\widetilde{A}_{1}$ is singular since so is $A\left(x_{0}\right)$. Further,

$$
\begin{aligned}
\widetilde{Q}_{1} & =\left[\begin{array}{ll}
Q\left(x_{0}\right) & 0 \\
Q\left(x_{0}\right) & 0
\end{array}\right] \quad \text { projects onto } \operatorname{ker}\left(\widetilde{A}_{1}\right), \\
\widetilde{P} \widetilde{P}_{1} & =\left[\begin{array}{cc}
P\left(x_{0}\right) & 0 \\
0 & 0
\end{array}\right], \quad \widetilde{A}_{2}=\widetilde{A}_{1}+\widetilde{B} \widetilde{P} \widetilde{Q}_{1}=\left[\begin{array}{cc}
I & -I \\
\mathcal{A} & A\left(x_{0}\right)
\end{array}\right],
\end{aligned}
$$

where $\mathcal{A}:=\left(A_{x}^{\prime}\left(x_{0}\right) y_{0}+g^{\prime}\left(x_{0}\right)\right) Q\left(x_{0}\right)$. 
The matrix $\widetilde{A}_{2}$ is nonsingular provided that so is $A_{1}\left(x_{0}, y_{0}\right)$. Hence $\{\widetilde{A}, \widetilde{B}\}$ is a regular index 2 pencil. Further, we compute

$$
\begin{aligned}
\widetilde{A}_{2}^{-1} & =\left[\begin{array}{cc}
I-A_{1}^{-1} \mathcal{A} & A_{1}^{-1} \\
-A_{1}^{-1} \mathcal{A} & A_{1}^{-1}
\end{array}\right], \quad A_{1}:=A_{1}\left(x_{0}, y_{0}\right), \\
\widetilde{P} \widetilde{P}_{1} \widetilde{A}_{2}^{-1} \widetilde{B} & =\left[\begin{array}{cc}
P\left(x_{0}\right) A_{1}^{-1}\left(A_{x}^{\prime}\left(x_{0}\right) y_{0}+g^{\prime}\left(x_{0}\right)\right) & 0 \\
0 & 0
\end{array}\right] .
\end{aligned}
$$

Theorem 2.5. Let $A(\cdot)$ and $g(\cdot)$ belong to the class $C^{1}$. For given $x_{0} \in \mathcal{D}$, $y_{0} \in \mathbb{R}^{m}$, let the matrix $A_{1}\left(x_{0}, y_{0}\right)$ given by $(2.20)$ be nonsingular; moreover, let

$$
\operatorname{im}(A(x))=\operatorname{im}\left(A\left(x_{0}\right)\right)
$$

for all $x$ from a neighbourhood of $x_{0}$. Additionally, let the consistency conditions

$$
A\left(x_{0}\right) y_{0}+g\left(x_{0}\right)=0, \quad A_{1}\left(x_{0}, y_{0}\right) y_{0}+g\left(x_{0}\right)=0
$$

be satisfied. Then

(i) The IVPs for (2.17) with the initial conditions

$$
\begin{aligned}
& P\left(x_{0}\right)\left(x\left(t_{0}\right)-x^{0}\right)=0, \quad x^{0} \in \mathbb{R}^{m}, \\
& \left|P\left(x_{0}\right)\left(x^{0}-x_{0}\right)\right| \leq \tau, \quad \tau \text { sufficiently small, }
\end{aligned}
$$

are solvable in $C^{1}$.

(ii) If , moreover, $A(\cdot)$ and $g(\cdot)$ are even $C^{2}$ functions, then the IVPs are uniquely solvable, and the solutions belong to $C^{2}$.

Proof. Condition (2.21) is nothing else but condition (2.13) for (2.19). Namely, we have here

$$
\widetilde{R}=\left(\begin{array}{ll}
I & 0 \\
0 & 0
\end{array}\right), \quad(I-\widetilde{R}) \widetilde{B} \widetilde{Q}=\left[\begin{array}{cc}
0 & 0 \\
0 & A\left(x_{0}\right)
\end{array}\right] .
$$

Thus we have to satisfy now

$$
A(x) y+g(x)-g(x) \in \operatorname{im}\left(A\left(x_{0}\right)\right), \quad \text { i.e. }(2.21) .
$$

The consistency conditions (2.22) are derived from (2.14) for (2.19). Hence, the statement is proved by applying Theorem 2.3 to the enlarged system (2.19).

R e m arks. 1. Clearly, Theorem 2.4 can also be applied to (2.19) in the same manner. If $x_{0}$ is now an equilibrium of (2.17), then the spectrum of the matrix

$$
-P\left(x_{0}\right) A_{1}^{-1}\left(A_{x}^{\prime}\left(x_{0}\right) y_{0}+g^{\prime}\left(x_{0}\right)\right)
$$

and the pencil

$$
\left\{A\left(x_{0}\right), A_{x}^{\prime}\left(x_{0}\right) y_{0}+g^{\prime}\left(x_{0}\right)\right\},
$$

is responsible for the stability behaviour.

2. Denote by $x_{*}(\cdot):\left[t_{0}, T\right] \rightarrow \mathbb{R}^{m}$ the solution of $(2.17)$ with $x_{*}\left(t_{0}\right)=x_{0}$ provided by Theorem 2.5(ii). Consider the perturbed IVPs

$$
A(x(t)) x^{\prime}(t)+g(x(t))=q(t), \quad P\left(x_{0}\right)\left(x\left(t_{0}\right)-x_{0}\right)=0 .
$$


Then, due to [7], Theorem 3.3 , the IVP $(2.23)$ has a unique $C^{1}$ solution $x(\cdot)$ : $\left[t_{0}, T\right] \rightarrow \mathbb{R}^{m}$, provided

$$
\|q\|_{\infty}+\left\|\left(Q\left(x_{0}\right) A_{1}^{-1} q\right)^{\prime}\right\|_{\infty}
$$

is sufficiently small. Moreover, we have

$$
\left\|x-x_{*}\right\|_{C^{1}} \leq K\left\{\|q\|_{\infty}+\left\|\left(Q\left(x_{0}\right) A_{1}^{-1} q\right)^{\prime}\right\|_{\infty}\right\} .
$$

3. The consistency conditions (2.22) are obviously equivalent to

$$
A\left(x_{0}\right) y_{0}+g\left(x_{0}\right)=0, \quad Q\left(x_{0}\right) y_{0}=0
$$

because of $A_{1}\left(x_{0}, y_{0}\right)^{-1} A\left(x_{0}\right)=P\left(x_{0}\right)$.

3. Index 3 case. In this section we try to obtain analogues of Theorems 2.3 and 2.4 for an appropriate class of index 3 DAEs.

We continue investigating the DAE (1.1). By Lemma 4.3 below, for fixed $x_{0} \in \mathcal{M}_{1}:=\left\{x \in \mathcal{D}: g\left(x_{0}\right) \in \operatorname{im}(A)\right\}$, we determine the matrices $A_{1}, A_{2}, A_{3}$, and the projector matrices $Q, Q_{1}, Q_{2}, P=I-Q, P_{1}=I-Q_{1}, P_{2}=I-Q_{2}$, according to the pencil

$$
\left\{A, g^{\prime}\left(x_{0}\right)\right\}=:\{A, B\}
$$

which is now assumed to be regular with index 3. Note that the pencil (3.1) is a regular index 3 pencil if and only if $A_{1}, A_{2}$ are singular but $A_{3}$ is nonsingular (see e.g. [4]).

We will use a similar technique of decoupling (1.1) by projections as in [7] for index 2 DAEs. To give a first insight into that procedure, let us briefly deal with the linear constant coefficient DAE

$$
A x^{\prime}(t)+B x(t)=q(t) .
$$

Due to Lemma 4.3 below, $(3.2)$ is equivalent to the system

$$
\left\{\begin{aligned}
\left(P P_{1} P_{2} x\right)^{\prime}+P P_{1} P_{2} A_{3}^{-1} B P P_{1} P_{2} x & =P P_{1} P_{2} A_{3}^{-1} q, \\
-\left(Q P_{1} Q_{2} x\right)^{\prime}-\left(Q Q_{1} x\right)^{\prime}+Q x & =Q P_{1} P_{2} A_{3}^{-1} q, \\
-\left(Q_{1} Q_{2} x\right)^{\prime}+Q_{1} x & =Q_{1} P_{2} A_{3}^{-1} q, \\
Q_{2} x & =Q_{2} A_{3}^{-1} q .
\end{aligned}\right.
$$

This is realized by scaling (3.2) by $A_{3}^{-1}$ and decomposing $I=P P_{1} P_{2}+Q P_{1} P_{2}+$ $Q_{1} P_{2}+Q_{2}$. Note that these products of projectors are projectors again.

The system shows clearly the different quality of the solution components: $P P_{1} P_{2} x$ solves a regular explicit ODE, $Q_{2} x$ is an "algebraic" component, computing $Q_{1} x$ includes a differentiation, and for the null space component certain components of $q$ have to be differentiated twice. We are going to take this into account by using the decomposition

$$
I=P P_{1} P_{2}+P P_{1} Q_{2}+P Q_{1}+Q
$$

for the solutions. Note that the products in (3.4) are also projectors. 
Rewriting the nonlinear DAE (1.1) as

$$
A x^{\prime}(t)+B\left(x(t)-x_{0}\right)+h(x(t))+g\left(x_{0}\right)=0,
$$

where $h(y):=g(y)-g\left(x_{0}\right)-g^{\prime}\left(x_{0}\right)\left(y-x_{0}\right), y \in \mathcal{D}$, and putting

$$
\begin{array}{rlrl}
u & :=P P_{1} P_{2} x, & v & :=Q_{2} x, \\
u_{0} & :=P P_{1} P_{2} x_{0}, \quad v_{0}:=Q_{2} x_{0}, \quad w_{0}:=Q_{1} x, \quad z:=Q x,
\end{array}
$$

we proceed with (3.5) as we did with (3.3) before. This leads to

$$
\begin{array}{r}
u^{\prime}+P P_{1} P_{2} A_{3}^{-1} B\left(u-u_{0}\right)+P P_{1} P_{2} A_{3}^{-1}\left(h\left(u+P P_{1} v+P w+z\right)+g\left(x_{0}\right)\right)=0, \\
-\left(Q P_{1} v\right)^{\prime}-(Q w)^{\prime}+z-z_{0}+Q P_{1} P_{2} A_{3}^{-1}\left(h\left(u+P P_{1} v+P w+z\right)+g\left(x_{0}\right)\right)=0, \\
-\left(Q_{1} v\right)^{\prime}+w-w_{0}+Q_{1} P_{2} A_{3}^{-1}\left(h\left(u+P P_{1} v+P w+z\right)+g\left(x_{0}\right)\right)=0, \\
v-v_{0}+Q_{2} A_{3}^{-1} h\left(u+P P_{1} v+P w+z\right)=0
\end{array}
$$

since $Q_{2} A_{3}^{-1} g\left(x_{0}\right)=0$.

Next we restrict the class of DAEs as for Lemma 2.2, aiming at decoupling the above nonlinear system. We formulate these restrictions in terms of the decoupling technique; they will be discussed below.

Let the conditions

$$
\begin{aligned}
Q_{2} A_{3}^{-1}\left\{g(y)-g\left(P P_{1} y\right)\right\} & =0, & & y \in U, \\
\left(Q_{1}+P P_{1}\right) P_{2} A_{3}^{-1}\{g(y)-g(P y)\} & =0, & & y \in U,
\end{aligned}
$$

be satisfied, where $U \subset \mathcal{D}$ denotes a neighbourhood of $x_{0}$. They lead to

$$
\begin{array}{rlrl}
Q_{2} A_{3}^{-1}\left\{h(y)-h\left(P P_{1} y\right)\right\} & =0, & & y \in U, \\
\left(Q_{1}+P P_{1}\right) P_{2} A_{3}^{-1}\{h(y)-h(P y)\} & =0, & y \in U,
\end{array}
$$

because of

$$
Q_{2} A_{3}^{-1} B=Q_{2} A_{3}^{-1} B P P_{1}, \quad\left(Q_{1}+P P_{1}\right) P_{2} A_{3}^{-1} B Q=0 .
$$

Due to the conditions (3.8), (3.9) the above system simplifies to

(3.10) $u^{\prime}+P P_{1} P_{2} A_{3}^{-1} B\left(u-u_{0}\right)+P P_{1} P_{2} A_{3}^{-1}\left(h\left(u+P P_{1} v+P w\right)+g\left(x_{0}\right)\right)=0$,

$$
\begin{aligned}
-\left(Q P_{1} v\right)^{\prime}-(Q w)^{\prime}+z-z_{0} & \\
+Q P_{1} P_{2} A_{3}^{-1}\left(h\left(u+P P_{1} v+P w+z\right)+g\left(x_{0}\right)\right) & =0, \\
-\left(Q_{1} v\right)^{\prime}+w-w_{0}+Q_{1} P_{2} A_{3}^{-1}\left(h\left(u+P P_{1} v+P w\right)+g\left(x_{0}\right)\right) & =0, \\
v-v_{0}+Q_{2} A_{3}^{-1} h\left(u+P P_{1} v\right) & =0 .
\end{aligned}
$$

Next we assume that $x_{0}$ is a consistent initial value, and that $x \in C_{N}^{1}$ solves the related IVP. Hence $u, v, w \in C^{1}, z \in C$ form a solution of (3.10)-(3.13) passing through $u_{0}, v_{0}, w_{0}, z_{0}$. Note that $h\left(x_{0}\right)=0, h^{\prime}\left(x_{0}\right)=0$, and $h$ is of class $C^{1}$.

Differentiating (3.13) we get

$$
v^{\prime}(t)+Q_{2} A_{3}^{-1} h^{\prime}\left(u(t)+P P_{1} v(t)\right)\left(u^{\prime}(t)+P P_{1} v^{\prime}(t)\right)=0,
$$


which leads to

$$
v^{\prime}\left(t_{0}\right)=0, \quad Q_{1} v^{\prime}\left(t_{0}\right)=0, \quad Q P_{1} v^{\prime}\left(t_{0}\right)=0 .
$$

Consequently, (3.12) is satisfied at $t=t_{0}$ if and only if the consistency condition

$$
Q_{1} P_{2} A_{3}^{-1} g\left(x_{0}\right)=0
$$

is satisfied.

From (3.12) it is clear that $Q_{1} v$ is of class $C^{2}$. Further, from (3.10) we know that so is $u$.

Consequently, also the related components of $h$ and $g$ should be $C^{2}$.

In the following we simply assume $Q_{2} A_{3}^{-1} g$ to be a $C^{2}$ function. Then so is $Q_{2} A_{3}^{-1} h$.

From (3.12), (3.13), (3.10) we derive

$$
\begin{aligned}
w^{\prime}\left(t_{0}\right) & =Q_{1} v^{\prime \prime}\left(t_{0}\right)=-Q_{1} Q_{2} A_{3}^{-1} h^{\prime \prime}\left(x_{0}\right) u^{\prime}\left(t_{0}\right) u^{\prime}\left(t_{0}\right) \\
& =-Q_{1} Q_{2} A_{3}^{-1} h^{\prime \prime}\left(x_{0}\right) P P_{1} P_{2} A_{3}^{-1} g\left(x_{0}\right) P P_{1} P_{2} A_{3}^{-1} g\left(x_{0}\right) .
\end{aligned}
$$

Now, an additional consistency condition follows from (3.11), namely

$$
Q P_{1} P_{2} A_{3}^{-1} g\left(x_{0}\right)=-Q Q_{1} Q_{2} A_{3}^{-1} g^{\prime \prime}\left(x_{0}\right) P P_{1} P_{2} A_{3}^{-1} g\left(x_{0}\right) P P_{1} P_{2} A_{3}^{-1} g\left(x_{0}\right) \text {. }
$$

Theorem 3.1. Let $g$ be a $C^{1}$ function, $x_{0} \in \mathcal{D}$ be fixed, $B:=g^{\prime}\left(x_{0}\right)$. Let the pencil $\{A, B\}$ be regular with index 3 , and let $Q, Q_{1}, Q_{2}, A_{1}, A_{2}, A_{3}$ be determined by Lemma 4.4 below. Let $Q_{2} A_{3}^{-1} g$ be a $C^{2}$ function, and the conditions (3.6), (3.7) be satisfied. Additionally, assume the consistency conditions (3.14), (3.15) and

$$
g\left(x_{0}\right) \in \operatorname{im}(A)
$$

to be satisfied. Then there is a $\tau>0$ such that all IVPs for (1.1) with initial conditions

$$
\begin{aligned}
P P_{1} P_{2}\left(x\left(t_{0}\right)-x^{0}\right) & =0, \quad x^{0} \in \mathbb{R}^{m}, \\
\left|P P_{1} P_{2}\left(x^{0}-x_{0}\right)\right| & \leq \tau
\end{aligned}
$$

are uniquely solvable in $C_{N}^{1}$.

Proof. For simplicity assume $x_{0}=0$ and $u_{0}=0, v_{0}=0, w_{0}=0, z_{0}=0$.

The system (3.10)-(3.13) gives the idea how to proceed. Motivated by (3.13) consider the $C^{2}$ function

$$
F(u, v):=v+Q_{2} A_{3}^{-1} h\left(u+P P_{1} v\right)
$$

acting from $\mathbb{R}^{m} \times \mathbb{R}^{m}$ into $\mathbb{R}^{m}$.

Because of $F(0,0)=0, F_{v}^{\prime}(0,0)=I$, the Implicit Function Theorem provides a $C^{2}$ function $f$ with

$$
\begin{aligned}
F(u, f(u)) & =0, \quad u \in \mathcal{B}\left(0, \varrho_{1}\right), \\
f(0) & =0, \quad f^{\prime}(0)=0, \\
f^{\prime \prime}(0) & =-F_{u u}^{\prime \prime}(0,0)=-Q_{2} A_{3}^{-1} h^{\prime \prime}(0)=-Q_{2} A_{3}^{-1} g^{\prime \prime}(0) .
\end{aligned}
$$


Next, if we supposed the IVP were solvable we would have (cf. (3.10))

$$
\begin{aligned}
v(t)= & f(u(t)), \\
v^{\prime}(t)= & -f^{\prime}(u(t))\left\{P P_{1} P_{2} A_{3}^{-1} B u(t)\right. \\
& \left.+P P_{1} P_{2} A_{3}^{-1}\left(h\left(u(t)+P P_{1} f(u(t))+P w(t)\right)+g(0)\right)\right\} .
\end{aligned}
$$

This fact, together with (3.12), motivates us to consider the $C^{1}$ function

$$
\begin{aligned}
& K(u, w):=w+Q_{1} P_{2} A_{3}^{-1} h\left(u+P P_{1} f(u)+P w\right) \\
& \quad+Q_{1} f^{\prime}(u)\left\{P P_{1} P_{2} A_{3}^{-1} B u+P P_{1} P_{2} A_{3}^{-1}\left(h\left(u+P P_{1} f(u)+P w\right)+g(0)\right)\right\}
\end{aligned}
$$

acting from $\mathbb{R}^{m} \times \mathbb{R}^{m}$ into $\mathbb{R}^{m}$. Since $K(0,0)=0, K_{w}^{\prime}(0,0)=I$, there is a $C^{1}$ function $k: \mathcal{B}\left(0, \varrho_{2}\right) \rightarrow \mathbb{R}^{m}$ such that

$$
k(0)=0, \quad K(u, k(u))=0, \quad u \in \mathcal{B}\left(0, \varrho_{2}\right) .
$$

Additionally, we derive

$$
\begin{aligned}
k^{\prime}(0) & =-K_{u}^{\prime}(0,0)=-Q_{1} f^{\prime \prime}(0) P P_{1} P_{2} A_{3}^{-1} g(0) \\
& =Q_{1} Q_{2} A_{3}^{-1} g^{\prime \prime}(0) P P_{1} P_{2} A_{3}^{-1} g(0) .
\end{aligned}
$$

Inserting $v=f(u), w=k(u)$ formally into (3.10) provides the regular ODE

$$
u^{\prime}+P P_{1} P_{2} A_{3}^{-1} B u+P P_{1} P_{2} A_{3}^{-1}\left(h\left(u+P P_{1} f(u)+P k(u)\right)+g(0)\right)=0 .
$$

Further, inspired by (3.11) consider the additional function

$$
\begin{aligned}
L(u, z):= & z+Q P_{1} P_{2} A_{3}^{-1}\left(h\left(u+P P_{1} f(u)+P k(u)+z\right)+g(0)\right) \\
& +\left(Q k^{\prime}(u)+Q P_{1} f^{\prime}(u)\right) P P_{1} P_{2} A_{3}^{-1}\{B u \\
& \left.+h\left(u+P P_{1} f(u)+P k(u)\right)+g(0)\right\} .
\end{aligned}
$$

$L$ is continuous and has a continuous partial Jacobian $L_{z}^{\prime}$. We have

$$
L(0,0)=Q P_{1} P_{2} A_{3}^{-1} g(0)+Q k^{\prime}(0) P P_{1} P_{2} A_{3}^{-1} g(0)=0
$$

according to condition (3.15); further, $L_{z}^{\prime}(0,0)=I$. Again the Implicit Function Theorem provides a continuous function $l: \mathcal{B}\left(0, \varrho_{3}\right) \rightarrow \mathbb{R}^{m}$ with $l(0)=0$, $L(u, l(u))=0, u \in \mathcal{B}\left(0, \varrho_{3}\right)$.

Next, return to the regular ODE (3.19). Multiplying it by $I-P P_{1} P_{2}$ we find

$$
\left(I-P P_{1} P_{2}\right) u^{\prime}=0, \quad \text { i.e. } \quad\left(\left(I-P P_{1} P_{2}\right) u\right)^{\prime}=0 .
$$

Therefore, choosing

$$
u\left(t_{0}\right)=P P_{1} P_{2} x^{0}
$$

we obtain an IVP-solution which does not leave the subspace $\operatorname{im}\left(P P_{1} P_{2}\right)$.

On the other hand, by construction,

$$
f(u)=Q_{2} f(u), \quad k(u)=Q_{1} k(u), \quad l(u)=Q l(u)
$$

for $u \in \mathcal{B}\left(0, \varrho_{4}\right), \varrho_{4}=\min \left\{\varrho_{1}, \varrho_{2}, \varrho_{3}\right\}$. 
Finally, the ODE (3.19) should be regarded on a region that is determined by $\mathcal{B}\left(0, \varrho_{4}\right)$ as well as by $U$ of the conditions (3.6), (3.7). Solving the IVP (3.19), (3.20) and putting

$$
x(t):=u(t)+P P_{1} f(u(t))+P k(u(t))+l(u(t)), \quad t \in \mathcal{I},
$$

we obtain a $C_{N}^{1}$ solution of (1.1), (3.17).

Rem arks. 1. Note that the solution components $P P_{1} P_{2} x, Q_{2} x$ are even $C^{2}$ functions.

2. If, additionally, $Q_{2} A_{3}^{-1} g \in C^{3},\left(P P_{1}+Q_{1}\right) P_{2} A_{3}^{-1} g \in C^{2}$, then $f \in C^{3}$, $k \in C^{2}$ and $l \in C^{1}$, hence $x \in C^{1}, P x \in C^{2}$.

3. The explicit ODE (3.19) represents the inherent local state system of (1.1).

4. Denote again by $R \in L\left(\mathbb{R}^{m}\right)$ a projector onto $\operatorname{im}(A)$. Then

$$
(I-R)\left\{g(y)-g\left(P P_{1} y\right)\right\} \in \operatorname{im}\left((I-R) B\left(Q+P Q_{1}\right)\right), \quad y \in U,
$$

is an equivalent formulation of (3.6). Roughly speaking, this requires the derivative free part in (1.1) to depend only linearly on the components $Q x, P Q_{1} x$.

5. Since $Q_{2} A_{3}^{-1} A=0$,

$$
(I-R) g \in C^{2} \quad \text { implies } \quad Q_{2} A_{3}^{-1} g \in C^{2} .
$$

Theorem 3.2. Let $g: \mathcal{D} \rightarrow \mathbb{R}^{m}$ be of class $C^{2}, x_{*} \in \mathcal{D}, g\left(x_{*}\right)=0, B:=g^{\prime}\left(x_{*}\right)$, let the pencil $\{A, B\}$ be regular of index 3 , and let all its eigenvalues have negative real parts. Further, let the conditions (3.6), (3.7) be satisfied on a neighbourhood $U$ of $x_{*}$.

Then there are $\tau>0$ and $\delta(\varepsilon)>0$ for each $\varepsilon>0$, such that

(i) all IVPs (1.1), (3.17), $\left|P P_{1} P_{2}\left(x^{0}-x_{*}\right)\right| \leq \tau$ have unique solutions defined on $\left[t_{0}, \infty\right)$,

(ii) $\left|P P_{1} P_{2}\left(x^{0}-x_{*}\right)\right| \leq \delta(\varepsilon)$ implies $\left|x\left(t ; x^{0}, t_{0}\right)-x_{*}\right| \leq \varepsilon$ for all $t \geq t_{0}$, and

(iii) $\left|x\left(t ; x^{0}, t_{0}\right)-x_{*}\right| \rightarrow 0(t \rightarrow \infty)$.

Proof. For more transparence, assume $x_{*}=0$. Since $g\left(x_{*}\right)=0$, the consistency conditions (3.14), (3.15), (3.16) are satisfied trivially, hence the IVPs (1.1), (3.17) have local $C_{N}^{1}$ solutions $x(\cdot): \mathcal{I} \rightarrow \mathbb{R}^{m}$. Then the components $u:=P P_{1} P_{2} x$ solve the regular IVPs (cf. (3.19))

$$
\begin{gathered}
u^{\prime}+P P_{1} P_{2} A_{3}^{-1} B u+P P_{1} P_{2} A_{3}^{-1} h\left(u+P P_{1} f(u)+P k(u)\right)=0, \\
u\left(t_{0}\right)=P P_{1} P_{2} x^{0}, \quad\left|P P_{1} P_{2} x^{0}\right| \leq \tau,
\end{gathered}
$$

and $x=u+P P_{1} f(u)+P k(u)+l(u)$.

Recall from Theorem 3.1 that $f \in C^{2}, k \in C^{1}, l \in C$.

By Lemma 4.5 below, the matrix $M:=-P P_{1} P_{2} A_{3}^{-1} B$ has the same nontrivial eigenvalues as the pencil $\{A, B\}$. The remaining eigenvalues of $M=P P_{1} P_{2} M=$ $M P P_{1} P_{2}$ are zero, and their structure is simple. Therefore, Lemma 4.4 below provides a scalar product which may be applied as a local Lyapunov function related to $\operatorname{im}\left(P P_{1} P_{2}\right)$ to the explicit ODE (3.23) in the standard way (see e.g. [6], Theorem 4.3). There, we take into consideration that $\mathcal{S}(u):=h\left(u+P P_{1} f(u)+\right.$ 
$P k(u))$ is continuously differentiable, $\mathcal{S}(0)=0, \mathcal{S}^{\prime}(0)=0$, and that, as is easy to check,

with small $\varrho$.

$$
|\mathcal{S}(u)| \leq c|u|^{2} \quad \text { for } u \in \mathcal{B}(0, \varrho)
$$

By Theorem 3.2, checking the spectrum of the pencil $\{A, B\}$ or, equivalently, that of the matrix $P P_{1} P_{2} A_{3}^{-1} B$, and proving the conditions (3.6), (3.7) to be satisfied, will do to know whether an equilibrium point $x_{*}$ of (1.1) is asymptotically stable.

For important classes of DAEs (1.1) the conditions (3.6), (3.7) are valid due to the special structure of those equations. We finish this paper by considering Euler-Lagrange formulations of constrained multibody systems in some more detail. Consider the nonlinear system

$$
\left\{\begin{aligned}
u^{\prime}-v & =0, \\
v^{\prime}+f(u, v)+h^{\prime}(u)^{\mathrm{T}} w & =0, \\
h(u) & =0 .
\end{aligned}\right.
$$

Assume $h^{\prime}(u)$ to have full rank, i.e. the holonomic constraints to be linearly independent. Note that $w$ represents the Lagrange multiplier, $u$ the position, and $v$ the velocity. For given $x_{0}^{\mathrm{T}}=\left(u_{0}^{\mathrm{T}}, v_{0}^{\mathrm{T}}, w_{0}^{\mathrm{T}}\right)$ we compute

$$
A=\left[\begin{array}{lll}
I & 0 & 0 \\
0 & I & 0 \\
0 & 0 & 0
\end{array}\right], \quad B=\left[\begin{array}{ccc}
0 & -I & 0 \\
F & G & H^{\mathrm{T}} \\
H & 0 & 0
\end{array}\right] \text {, }
$$

where $F:=f_{u}^{\prime}\left(u_{0}, v_{0}\right)+h^{\prime \prime}\left(u_{0}\right)^{\mathrm{T}} w_{0}, G:=f_{v}^{\prime}\left(u_{0}, v_{0}\right), H:=h^{\prime}\left(u_{0}\right)$. Since $H$ has full rank, $H H^{\mathrm{T}}$ is nonsingular. Define $P_{H}:=H^{+} H, Q_{H}:=I-H^{+} H$, $H^{+}=H^{\mathrm{T}}\left(H H^{\mathrm{T}}\right)^{-1}$. Choosing

$$
\begin{gathered}
Q=\left[\begin{array}{ccc}
0 & 0 & 0 \\
0 & 0 & 0 \\
\alpha & \beta & I
\end{array}\right], \\
\alpha:=\left(H H^{\mathrm{T}}\right)^{-1} H\left\{F Q_{H}+G Q_{H} G P_{H}\right\}, \quad \beta:=\left(H H^{\mathrm{T}}\right)^{-1} H G Q_{H},
\end{gathered}
$$

we derive

$$
\begin{aligned}
A_{1} & =A+B Q=\left[\begin{array}{ccc}
I & 0 & 0 \\
H^{\mathrm{T}} \alpha & I+H^{\mathrm{T}} \beta & H^{\mathrm{T}} \\
0 & 0 & 0
\end{array}\right], \\
B_{1} & =B P=\left[\begin{array}{ccc}
0 & -I & 0 \\
F-H^{\mathrm{T}} \alpha & G-H^{\mathrm{T}} \beta & 0 \\
H & 0 & 0
\end{array}\right], \\
\operatorname{ker}\left(A_{1}\right) & =\left\{\left(u^{\mathrm{T}}, v^{\mathrm{T}}, w^{\mathrm{T}}\right)^{\mathrm{T}}: u=0, v+H^{\mathrm{T}} \beta v+H^{\mathrm{T}} w=0\right\} \\
& =\left\{\left(u^{\mathrm{T}}, v^{\mathrm{T}}, w^{\mathrm{T}}\right)^{\mathrm{T}}: u=0, v=P_{H} v, w=-\left(H H^{\mathrm{T}}\right)^{-1} H v\right\} ;
\end{aligned}
$$


further,

$$
\begin{aligned}
& Q_{1}=\left[\begin{array}{ccc}
0 & 0 & 0 \\
0 & P_{H} & 0 \\
0 & -\left(H H^{\mathrm{T}}\right)^{-1} H & 0
\end{array}\right] \\
& A_{2}=A_{1}+B_{1} Q_{1}=\left[\begin{array}{ccc}
I & -P_{H} & 0 \\
H^{\mathrm{T}} \alpha & I+H^{\mathrm{T}} \beta+G P_{H} & H^{\mathrm{T}} \\
0 & 0 & 0
\end{array}\right], \\
& \operatorname{ker}\left(A_{2}\right)=\left\{\left(u^{\mathrm{T}}, v^{\mathrm{T}}, w^{\mathrm{T}}\right)^{\mathrm{T}}: u=P_{H} u, v=P_{H} u-Q_{H} G P_{H} u,\right. \\
& \left.w=-\left(H H^{\mathrm{T}}\right)^{-1}\left(H u+H G P_{H} u\right)\right\}, \\
& S_{2}=\left\{\left(u^{\mathrm{T}}, v^{\mathrm{T}}, w^{\mathrm{T}}\right)^{\mathrm{T}}: H u=0\right\}, \\
& Q_{2}=\left[\begin{array}{ccc}
P_{H} & 0 & 0 \\
P_{H}-Q_{H} G P_{H} & 0 & 0 \\
-\left(H H^{\mathrm{T}}\right)^{-1} H\left(I+G P_{H}\right) & 0 & 0
\end{array}\right], \\
& A_{3}=A_{2}+B_{1} P_{1} Q_{2} \\
& =\left[\begin{array}{ccc}
I+Q_{H} G P_{H} & -P_{H} & 0 \\
H^{\mathrm{T}} \alpha+F P_{H}-G Q_{H} G P_{H} & I+H^{\mathrm{T}} \beta-G P_{H} & H^{\mathrm{T}} \\
H & 0 & 0
\end{array}\right] .
\end{aligned}
$$

Then $A_{3}$ is nonsingular, $A_{3}^{-1}$ equals

$$
\left[\begin{array}{ccc}
Q_{H} & 0 & \left(I-Q_{H} G\right) H^{+} \\
-P_{H}+Q_{H} G P_{H} & Q_{H} & \left(I-Q_{H} G-Q_{H} F+Q_{H} G Q_{H} G\right) H^{+} \\
\gamma & \left(H H^{\mathrm{T}}\right)^{-1} H\left(I-G Q_{H}\right) & \delta
\end{array}\right] \text {, }
$$

where

$$
\begin{aligned}
\gamma:= & \left(H H^{\mathrm{T}}\right)^{-1} H\left(I+G P_{H}-G Q_{H} G P_{H}-F Q_{H}\right), \\
\delta:= & \left(H H^{\mathrm{T}}\right)^{-1} H\left(-I-G-F+G Q_{H} G+G Q_{H} F\right. \\
& \left.+F Q_{H} G-G Q_{H} G Q_{H} G\right) H^{+} .
\end{aligned}
$$

Moreover, it may be checked that, in fact,

$$
Q=Q P_{1} P_{2} A_{3}^{-1} B, \quad Q_{1}=Q_{1} P_{2} A_{3}^{-1} B P, \quad Q_{2}=Q_{2} A_{3}^{-1} B P P_{1}
$$

are the projectors announced in Lemma 4.3 below.

Furthermore, we have

$$
\begin{gathered}
P=\left[\begin{array}{ccc}
I & 0 & 0 \\
0 & I & 0 \\
-\alpha & -\beta & 0
\end{array}\right], \quad P P_{1}=\left[\begin{array}{ccc}
I & 0 & 0 \\
0 & Q_{H} & 0 \\
-\alpha & -\beta & 0
\end{array}\right], \\
\text { and } P P_{1} P_{2}=\left[\begin{array}{ccc}
Q_{H} & 0 & 0 \\
Q_{H} G P_{H} & Q_{H} & 0 \\
-\alpha & -\beta & 0
\end{array}\right] .
\end{gathered}
$$

Are the conditions (3.6), (3.7) satisfied? Because of 


$$
\begin{aligned}
\left(Q_{1}+P P_{1}\right) P_{2} A_{3}^{-1}\{g(x)- & g(P x)\} \\
= & \left(\begin{array}{c}
0 \\
Q_{H} h^{\prime}(u)^{\mathrm{T}}(w+\alpha u+\beta v) \\
-\beta h^{\prime}(u)^{\mathrm{T}}(w+\alpha u+\beta v)
\end{array}\right), \quad x=\left(\begin{array}{c}
u \\
v \\
w
\end{array}\right),
\end{aligned}
$$

condition (3.7) is satisfied for all $x$ from a neighbourhood $U$ of $x_{0}$, provided

$$
Q_{H} h^{\prime}(u)^{\mathrm{T}}=0
$$

for the component $u$, i.e.

$$
\operatorname{ker}\left(h^{\prime}(u)\right)=\operatorname{ker}\left(h^{\prime}\left(u_{0}\right)\right) .
$$

On the other hand, for $x \in U$,

$$
\begin{aligned}
Q_{2} A_{3}^{-1}\left\{g(x)-g\left(P P_{1} x\right)\right\} & =\left[\begin{array}{ccc}
0 & 0 & H^{+} \\
0 & 0 & \left(I-Q_{H} G\right) H^{+} \\
0 & 0 & -\left(H H^{\mathrm{T}}\right)^{-1} H(I+G) H^{+}
\end{array}\right] \\
& \times\left[\begin{array}{c}
h^{\prime}(u)^{\mathrm{T}}(w+\alpha u+\beta v)+f(u, v)-f\left(u, Q_{H} v\right) \\
0
\end{array}\right]=0 .
\end{aligned}
$$

Next, consider the consistency conditions (3.14), (3.15), (3.16) applied to the system (3.25). Formula (3.16) can be simplified to

$$
h\left(u_{0}\right)=0 .
$$

Condition (3.14) means now

$$
\begin{aligned}
0= & {\left[\begin{array}{ccc}
0 & 0 & 0 \\
-P_{H} & P_{H} & 0 \\
\left(H H^{\mathrm{T}}\right)^{-1} H & \left(H H^{\mathrm{T}}\right)^{-1} H & 0
\end{array}\right] } \\
& \times\left[\begin{array}{c}
P_{H} v_{0}-Q_{H} G P_{H} v_{0}+Q_{H} f\left(u_{0}, v_{0}\right) \\
-\gamma v_{0}+\left(H H^{\mathrm{T}}\right)^{-1} H\left(I-G Q_{H}\right)\left(f\left(u_{0}, v_{0}\right)+h^{\prime}\left(v_{0}\right)^{\mathrm{T}} w_{0}\right)
\end{array}\right],
\end{aligned}
$$

that is, $P_{H} v_{0}=0$.

Further, it follows that

$$
\begin{aligned}
P P_{1} P_{2} A_{3}^{-1} g\left(x_{0}\right)= & {\left[\begin{array}{c}
-Q_{H} v_{0} \\
-Q_{H} G Q_{H} v_{0}+Q_{H} f\left(u_{0}, v_{0}\right) \\
-\alpha Q_{H} v_{0}-\beta Q_{H} f\left(u_{0}, v_{0}\right)
\end{array}\right], } \\
Q P_{1} P_{2} A_{3}^{-1} g\left(x_{0}\right)= & {\left[\begin{array}{c}
0 \\
0 \\
\left(H H^{\mathrm{T}}\right)^{-1} H\left\{f\left(u_{0}, v_{0}\right)+h^{\prime}\left(u_{0}\right)^{\mathrm{T}} w_{0}\right\}
\end{array}\right], } \\
Q Q_{1} Q_{2} A_{3}^{-1}= & {\left[\begin{array}{ccc}
0 & 0 & 0 \\
0 & 0 & 0 \\
-\left(H H^{\mathrm{T}}\right)^{-1} H & 0 & 0
\end{array}\right] A_{3}^{-1}=\left[\begin{array}{ccc}
0 & 0 & 0 \\
0 & 0 & 0 \\
0 & 0 & -\left(H H^{\mathrm{T}}\right)^{-1}
\end{array}\right], }
\end{aligned}
$$




$$
Q Q_{1} Q_{2} A_{3}^{-1} g^{\prime \prime}(x) y y=\left[\begin{array}{c}
0 \\
0 \\
-\left(H H^{\mathrm{T}}\right)^{-1} h^{\prime \prime}(u) y_{1} y_{1}
\end{array}\right],
$$

and, since the last component of $P P_{1} P_{2} A_{3}^{-1} g\left(x_{0}\right)$ belongs to $\operatorname{im}\left(Q_{H}\right)$,

$$
Q Q_{1} Q_{2} A_{3}^{-1} g^{\prime \prime}\left(x_{0}\right) P P_{1} P_{2} A_{3}^{-1} g\left(x_{0}\right) P P_{1} P_{2} A_{3}^{-1} g\left(x_{0}\right)=0
$$

has to be true. Consequently, condition (3.15) is satisfied if

$$
P_{H} f\left(u_{0}, v_{0}\right)+h^{\prime}\left(u_{0}\right)^{\mathrm{T}} w_{0}=0 .
$$

Now we summarize what we know about (3.25).

Corollary 3.3 Given the system (3.25) with $f \in C^{1}, h \in C^{2}$, and $u_{0}, v_{0}, w_{0}$ with

$$
h\left(u_{0}\right)=0, \quad P_{H} v_{0}=0, \quad P_{H} f\left(u_{0}, v_{0}\right)+h^{\prime}\left(u_{0}\right)^{\mathrm{T}} w_{0}=0 .
$$

Additionally, suppose

$$
\operatorname{ker}\left(h^{\prime}(u)\right)=\operatorname{ker}\left(h^{\prime}\left(u_{0}\right)\right)
$$

in a neighbourhood of $u_{0}$. Then

(i) Then the IVPs for (3.25) with

$$
Q_{H}\left(u\left(t_{0}\right)-u^{0}\right)=0, \quad Q_{H}\left(v\left(t_{0}\right)-v^{0}\right)=0
$$

are uniquely solvable in $C_{N}^{1}$, provided $\left|Q_{H}\left(u^{0}-u_{0}\right)\right|$ and $\left|Q_{H}\left(v^{0}-v_{0}\right)\right|$ are sufficiently small.

(ii) If, additionally, $f \in C^{2}, h \in C^{3}, Q_{H} v_{0}=0, Q_{H} f\left(u_{0}, v_{0}\right)=0$, and

$$
\operatorname{det}\left[\begin{array}{ccc}
\lambda I & -I & 0 \\
F & \lambda I+G & H^{\mathrm{T}} \\
H & 0 & 0
\end{array}\right]=0 \quad \text { implies } \quad \operatorname{Re} \lambda<0,
$$

then $\left(u_{0}^{\mathrm{T}}, v_{0}^{\mathrm{T}}, w_{0}^{\mathrm{T}}\right)^{\mathrm{T}}$ is an asymptotically stable equilibrium of (3.25).

Proof. These statements are specifications of Theorems 3.1 and 3.2, respectively. Thereby, $Q_{2} A_{3}^{-1} g$ is $C^{2}$ since so is $h$.

The initial condition (3.27) is equivalent to $P P_{1} P_{2}\left(x\left(t_{0}\right)-x^{0}\right)=0$.

\section{Appendix: Some linear algebra}

Lemma 4.1. Let $A, B, Q \in L\left(\mathbb{R}^{m}\right)$ be given, $Q^{2}=Q, \operatorname{im}(Q)=\operatorname{ker}(A)$, i.e. $Q$ projects $\mathbb{R}^{m}$ onto $\operatorname{ker}(A)$. Put $S:=\left\{z \in \mathbb{R}^{m}: B z \in \operatorname{im}(A)\right\}$. Then

(i) The decomposition $\mathbb{R}^{m}=S \oplus \operatorname{ker}(A)$ holds if and only if the matrix $G:=A+B Q$ is nonsingular for any projector $Q$ onto $\operatorname{ker}(A)$.

(ii) $\mathbb{R}^{m}=S \oplus \operatorname{ker}(A)$ implies $G^{-1} A=P, Q=G^{-1} B Q$, and $Q G^{-1} B$ represents the projector onto $\operatorname{ker}(A)$ along $S$.

Pro of. [3], Theorem A.13, Lemma A.14. 
Lemma 4.2. Let $\{A, B\}$ be a regular index 2 matrix pencil, $A, B \in L\left(\mathbb{R}^{m}\right)$. Then the projectors $Q, Q_{1} \in L\left(\mathbb{R}^{m}\right)$ may be chosen in such a way that

$$
Q=Q P_{1} A_{2}^{-1} B, \quad Q_{1}=Q_{1} A_{2}^{-1} B P,
$$

$Q$ projects onto $\operatorname{ker}(A)$,

$$
A_{1}:=A+B Q, \quad A_{2}:=A_{1}+B P Q_{1}, \quad P_{1}:=I-Q_{1},
$$

and $Q_{1}$ projects onto $\operatorname{ker}\left(A_{1}\right)$ along

$$
S_{1}:=\left\{z \in \mathbb{R}^{m}: B P z \in \operatorname{im}\left(A_{1}\right)\right\} .
$$

Proof. [7], Lemma 2.1.

Lemma 4.3. Let $\{A, B\}$ be a regular index 3 matrix pencil. Then the projectors $Q, Q_{1}, Q_{2} \in L\left(\mathbb{R}^{m}\right)$ may be chosen in such a way that

$$
Q_{2}=Q_{2} A_{3}^{-1} B P P_{1}, \quad Q_{1}=Q_{1} P_{2} A_{3}^{-1} B P, \quad Q=Q P_{1} P_{2} A_{3}^{-1} B,
$$

where $A_{1}:=A+B Q, A_{2}:=A_{1}+B P Q_{1}, A_{3}:=A_{2}+B P P_{1} Q_{2}, P:=I-Q$, $P_{i}=I-Q_{i}, i=1,2$,

$Q$ projects onto $\operatorname{ker}(A), \quad Q_{1}$ projects onto $\operatorname{ker}\left(A_{1}\right)$,

$Q_{2}$ projects onto $\operatorname{ker}\left(A_{2}\right)$ along $S_{2}:=\left\{z \in \mathbb{R}^{m}: B P P_{1} z \in \operatorname{im}\left(A_{2}\right)\right\}$.

Proof. Choose any projector $Q \in L\left(\mathbb{R}^{m}\right)$ onto $\operatorname{ker}(A)$ and put

$$
A_{1}:=A+B Q, \quad A_{2}:=A_{1}+B P Q_{1}, \quad A_{3}:=A_{2}+B P P_{1} Q_{2}
$$

where $Q_{1}, Q_{2} \in L\left(\mathbb{R}^{m}\right)$ are any projectors onto $\operatorname{ker}\left(A_{1}\right)$ and $\operatorname{ker}\left(A_{2}\right)$, respectively.

Due to [4], Theorem $3, A_{1}, A_{2}$ are singular but $A_{3}$ is not. By [4], Theorem 5 , we are allowed to choose $Q_{1}$ in such a way that $Q_{1} Q=0$. Moreover, we may choose $Q_{2}$ to project onto $\operatorname{ker}\left(A_{2}\right)$ along $S_{2}:=\left\{z \in \mathbb{R}^{m}: B P P_{1} z \in \operatorname{im}\left(A_{2}\right)\right\}$ by applying Lemma 4.1. Thus, $Q_{2}=Q_{2} A_{3}^{-1} B P P_{1}$.

Next, it is easy to check that

$$
\bar{Q}:=Q P_{1} P_{2} A_{3}^{-1} B
$$

is a projector onto $\operatorname{ker}(A)$.

Now, we start the above procedure again, by putting

$$
\bar{A}_{1}=A+B \bar{Q} .
$$

Taking into account that $\bar{Q}=Q \bar{Q}, Q=\bar{Q} Q$, we evaluate

$$
\bar{A}_{1}=A_{1}\left(I+Q P_{1} P_{2} A_{3}^{-1} B P\right),
$$

where $F_{1}:=I+Q P_{1} P_{2} A_{3}^{-1} B P$ is nonsingular, $F_{1}^{-1}=I-Q P_{1} P_{2} A_{3}^{-1} B P$. Hence $\operatorname{im}\left(\bar{A}_{1}\right)=\operatorname{im}\left(A_{1}\right)$.

Furthermore, $Q_{1} P_{2} A_{3}^{-1} B P$ is a projector onto $\operatorname{ker}\left(A_{1}\right)$, and thus $\bar{Q}_{1}:=$ $F_{1}^{-1} Q_{1} P_{2} A_{3}^{-1} B P F_{1}$ projects onto $\operatorname{ker}\left(\bar{A}_{1}\right)$. However,

$$
\bar{Q}_{1}=Q_{1} P_{2} A_{3}^{-1} B P,
$$

so that $\operatorname{ker}\left(A_{1}\right)=\operatorname{ker}\left(\bar{A}_{1}\right), \bar{Q}_{1} Q_{1}=Q_{1}$. 
Next compute

$$
\bar{A}_{2}:=\bar{A}_{1}+B \bar{P} \bar{Q}_{1}=A_{2} F_{2},
$$

where

$$
\begin{aligned}
F_{2} & :=F_{1}+Q_{1} P_{2} A_{3}^{-1} B P P_{1} \quad \text { is nonsigular, } \\
F_{2}^{-1} & =I-Q P_{1} P_{2} A_{3}^{-1} B P-Q_{1} P_{2} A_{3}^{-1} B P P_{1} .
\end{aligned}
$$

We observe that $\operatorname{im}\left(\bar{A}_{2}\right)=\operatorname{im}\left(A_{2}\right)$.

Since $\bar{Q}_{2}:=F_{2}^{-1} Q_{2} F_{2}=Q_{2}$, we also have $\operatorname{ker}\left(\bar{A}_{2}\right)=\operatorname{ker}\left(A_{2}\right)$. Moreover,

$$
S_{1}=\bar{S}_{1}, \quad S_{2}=\bar{S}_{2},
$$

and $\bar{A}_{3}=A_{3} F_{2}$.

Because of $S_{2}=\bar{S}_{2}, \operatorname{ker}\left(A_{2}\right)=\operatorname{ker}\left(\bar{A}_{2}\right), \bar{Q}_{2}$ represents the projector onto $\operatorname{ker}\left(\bar{A}_{2}\right)$ along $\bar{S}_{2}$. Due to Lemma 4.1,

$$
\bar{Q}_{2}=\bar{Q}_{2} \bar{A}_{3}^{-1} B \bar{P} \bar{P}_{1} .
$$

Further, it may be proved that

$$
\bar{Q}_{1}=\bar{Q}_{1} \bar{P}_{2} \bar{A}_{3}^{-1} B \bar{P} .
$$

Does $\bar{Q}=\bar{Q} \bar{P}_{1} \bar{P}_{2} \bar{A}_{3}^{-1} B$ hold? Unfortunately, it does not in general. We derive

$$
\bar{Q} \bar{P}_{1} \bar{P}_{2} \bar{A}_{3}^{-1} B=\bar{Q} \bar{P}_{1} \bar{P}_{2} \bar{A}_{3}^{-1} B \bar{P}+\bar{Q} \bar{P}_{1} \bar{P}_{2} \bar{A}_{3}^{-1} B \bar{Q}=\bar{Q} \bar{P}_{1} \bar{P}_{2} \bar{A}_{3}^{-1} B \bar{P}+\bar{Q},
$$

but

$$
\bar{Q} \bar{P}_{1} \bar{P}_{2} \bar{A}_{3}^{-1} B \bar{P}=Q\left(\bar{Q}_{1}-Q_{1}\right) P_{2} A_{3}^{-1} B P P_{1} P_{2}
$$

may not vanish.

Finally, start our procedure once more putting

$$
\overline{\bar{Q}}:=\bar{Q} \bar{P}_{1} \bar{P}_{2} \bar{A}_{3}^{-1} B .
$$

By the same arguments as above, we obtain

$$
\overline{\bar{Q}}_{1}=\overline{\bar{Q}}_{1} \overline{\bar{P}}_{2} \overline{\bar{A}}_{3}^{-1} B \overline{\bar{P}}=\bar{Q}_{1}, \quad \overline{\bar{Q}}_{2}=\overline{\bar{Q}}_{2} \overline{\bar{A}}_{3}^{-1} B \overline{\bar{P}}_{\bar{P}}=\bar{Q}_{2}=Q_{2},
$$

but now $\bar{Q}\left(\overline{\bar{Q}}_{1}-\bar{Q}_{1}\right) \bar{P}_{2} A_{3}^{-1} B \bar{P} \bar{P}_{1} \bar{P}_{2}=0$, and thus

$$
\overline{\bar{Q}} \overline{\bar{P}}_{1} \overline{\bar{P}}_{2} \overline{\bar{A}}_{3}^{-1} B=\overline{\bar{Q}} \text {. }
$$

Consequently, $\overline{\bar{Q}}, \overline{\bar{Q}}_{1}, \overline{\bar{Q}}_{2}$ are the projectors required.

LEMmA 4.4. Given $M, \Pi \in L\left(\mathbb{R}^{m}\right), \Pi^{2}=\Pi, M=\Pi M=M \Pi, \operatorname{rank}(\Pi)=: \mu$. Let $M$ have $\mu$ nontrivial eigenvalues $\lambda_{1}, \ldots, \lambda_{\mu}$, each with a negative real part. Then there is a constant $\beta>0$ and a regular matrix $C \in L\left(\mathbb{C}^{m}\right)$ such that

$$
\operatorname{Re}\langle M z, z\rangle_{C} \leq-\beta|z|_{C}^{2}, \quad z \in \operatorname{im}(\Pi),
$$

where $\langle u, v\rangle_{C}:=\left\langle C^{-1} u, C^{-1} v\right\rangle_{2}$.

Proof. [7], Lemma 4.2 . 
Lemma 4.5. Given a regular index 3 matrix pencil $\{A, B\}$. Let $Q, Q_{1}, Q_{2}$ be determined by Lemma 4.3, and

$$
M:=-P P_{1} P_{2} A_{3}^{-1} B, \quad \mu:=\operatorname{rank}\left(P P_{1} P_{2}\right) .
$$

Then

$$
\operatorname{grad} \operatorname{det}(\lambda A+B)=\mu
$$

and $\{A, B\}$ has $\mu$ eigenvalues, say $\lambda_{1}, \ldots, \lambda_{\mu}$.

Moreover, $\lambda_{1}, \ldots, \lambda_{\mu}$ also belong to the spectrum of $M$, where the corresponding eigenvectors lie in $\operatorname{im}\left(P P_{1} P_{2}\right)$. The remaining eigenvalues of $M$ are zero, and the corresponding eigenvectors span $\operatorname{ker}\left(P P_{1} P_{2}\right)$.

Proof. Note that $M=M P P_{1} P_{2}=P P_{1} P_{2} M$. Suppose $(\lambda A+B) w=0$, $w \neq 0$. Multiplying by $P P_{1} P_{2} A_{3}^{-1}, Q P_{1} P_{2} A_{3}^{-1}, Q_{1} P_{2} A_{3}^{-1}, Q_{2} A_{3}^{-1}$ yields

$$
\left\{\begin{aligned}
\lambda P P_{1} P_{2} w-M w & =0, \\
\lambda\left(-Q Q_{1}-Q P_{1} Q_{2}\right) w+Q w & =0, \\
\lambda\left(-Q_{1} Q_{2}\right) w+Q_{1} w & =0, \\
Q_{2} w & =0,
\end{aligned}\right.
$$

hence $w=P P_{1} P_{2} w, M w=\lambda w$.

On the other hand, suppose

$$
M z=\lambda z, \quad z \neq 0 .
$$

If $\lambda \neq 0$, then $M=P P_{1} P_{2} M$ yields $z=P P_{1} P_{2} z \neq 0$ immediately. In this case we have

$$
\begin{aligned}
\lambda P P_{1} P_{2} z & =-P P_{1} P_{2} A_{3}^{-1} B P P_{1} P_{2} z=-P_{1} P_{2} A_{3}^{-1} B P P_{1} P_{2} z \\
& =-P_{2} A_{3}^{-1} B P P_{1} P_{2} z=-A_{3}^{-1} B P P_{1} P_{2} z,
\end{aligned}
$$

since $Q P_{1} P_{2} A_{3}^{-1} B P=0, Q_{1} P_{2} A_{3}^{-1} B P P_{1}=0$ and $Q_{2} A_{3}^{-1} B P P_{1} P_{2}=0$. Hence $\lambda A_{3} P P_{1} P_{2} z+B P P_{1} P_{2} z=0$, i.e. $(\lambda A+B) P P_{1} P_{2} z=0$.

If $\lambda=0$ but $P P_{1} P_{2} z \neq 0$, we conclude as above that

$$
0=M z=-P P_{1} P_{2} A_{3}^{-1} B P P_{1} P_{2} z=-A_{3}^{-1} B P P_{1} P_{2} z,
$$

thus $B P P_{1} P_{2} z=0$.

Finally, $\operatorname{ker}\left(P P_{1} P_{2}\right) \subseteq \operatorname{ker}(M)$, and $z \in \operatorname{ker}\left(P P_{1} P_{2}\right), z \in \operatorname{im}(M) \subseteq \operatorname{im}\left(P P_{1} P_{2}\right)$ imply $z=0$, hence the related eigenstructure of $M$ is simple.

\section{References}

[1] L. O. Chua and A.-C. Deng, Impasse points. Part I: Numerical aspects, Internat. J. Circuit Theory Appl. 17 (1989), 213-235.

[2] C. W. Gear and L. R. Petzold, ODE methods for the solution of differential/algebraic systems, SIAM J. Numer. Anal. 21 (1984), 716-728.

[3] E. Griepentrog and R. März, Differential-Algebraic Equations and Their Numerical Treatment, Teubner-Texte Math. 88, Leipzig 1986. 
[4] E. Griepentrog and R. März, Basic properties of some differential-algebraic equations, Z. Anal. Anwendungen 8 (1989), 25-40.

[5] R. März, Index-2 differential-algebraic equations, Results in Math. 15 (1989), 149-171.

[6] - Some new results concerning index-3 differential-algebraic equations, J. Math. Anal. Appl. 140 (1989), 177-199.

[7] -, On quasilinear index 2 differential algebraic equations, preprint 269, Fachbereich Mathematik, Humboldt-Univ. Berlin, 1990.

[8] P. J. Rabier and W. Rheinboldt, A general existence and uniqueness theory for implicit differential-algebraic equations, Differential Integral Equations 4 (1991), 563-582. 